\title{
A SIMPLE FORMULA FOR PREDICTING THE FIRST NATURAL FREQUENCY OF TRANSVERSE VIBRATIONS OF AXIALLY LOADED HELICAL SPRINGS
}

\author{
Krzysztof Michalczyk, Piotr Bera \\ AGH University of Science and Technology, Faculty of Mechanical Engineering and Robotics, Cracow, Poland \\ e-mail:kmichal@agh.edu.pl; pbera@agh.edu.pl
}

\begin{abstract}
A new formula that allows the first natural frequency of transverse vibrations of axially loaded steel helical springs to be determined has been presented in the paper. The relationship is easy to use and allows finding the first natural frequency of spring vibrations without the necessity of solving analytical or numerical models. According to the authors' knowledge, this is the first such a formula and, consequently, when this frequency becomes zero, it enables determination of the critical axial force or deflection causing the buckling of the spring. The way of obtaining the described formula is presented in the paper. The results of this formula are compared with those obtained using FEM and experiments. The advantages, drawbacks and limitations of the proposed relationship are also discussed.
\end{abstract}

Keywords: helical springs, natural frequencies, helical spring vibrations, transverse vibrations, helical spring stability

\section{Introduction}

Springs can be treated as massless force elements with constant stiffness only at low excitation frequencies (Lee and Thompson, 2001). This simple model is often used in works concerning the analysis of low frequency vibrating machines or vibration reduction systems, e.g. (Cieplok, 2009; Sapiński et al., 2011). However, this model is not suitable for vibrations at higher frequencies, when there is a risk of excitation of spring resonance vibrations. It is then necessary to determine the natural frequencies of such a spring in order to avoid the spring surge phenomenon. In the case of cylindrical helical springs with a constant lead angle, the determination of natural frequencies of longitudinal vibrations does not pose a significant problem, because these frequencies can be estimated based on the well-known formula

$$
f_{L}=\frac{n}{2} \sqrt{\frac{k}{m_{S}}}
$$

where: $k$ - spring stiffness in $\mathrm{N} / \mathrm{m}$ and $m_{s}$ - spring mass in $\mathrm{kg}$, whereas the values of the coefficient $n$ equal to $1,2,3, \ldots$ refer to the numbers of subsequent free longitudinal vibration frequencies of a clamped-clamped spring. Such a model can be applied in a typical case, when one end of the spring is supported on the base and the second one is mounted to the object (e.g. machine) with a mass much greater than that of the spring. An analogical formula allowing the natural frequencies of transverse vibrations of axially loaded clamped-clamped helical springs to be determined does not exist in the available literature. The reason for that is the complexity of the analytical models describing this problem and the necessity of applying numerical methods to solve them. The development of such a formula would be very useful from the point of view of designers and researchers developing or analyzing dynamic systems in which helical springs are applied. Such a relationship could be widely used in engineering practice, because it would 
not require the knowledge of the methods for solving relatively complex analytical or numerical models, and the ease of its use would compensate its approximate character.

The aim of this study is, therefore, elaboration of a formula enabling estimation of the first natural frequency of transverse vibrations of clamped-clamped springs loaded with a static axial force. In the first stage of the research, the model of the Timoshenko type equivalent beam modified in (Michalczyk, 2015a) is utilized to calculate the values of the natural transverse vibration frequencies of steel helical springs for a wide range of geometrical parameters and relative axial deflections. In the second stage of the research, the obtained data set is utilized in formulation of the relationship allowing estimation of the first natural transverse vibration frequencies of axially loaded clamped-clamped steel springs of arbitrary - within a defined range - geometrical parameters and the relative axial deflection.

\section{Analytical model of helical spring transverse vibrations}

In the papers where free vibrations of helical springs are investigated, three types of models allowing the parameters of these vibrations to be determined are utilized. The first one is the model in which the spring, which is actually a continuous system, is replaced by a periodic discrete system in which the coils or their parts are replaced by masses connected by massless elastic elements. Such a model is often applied in calculations of free longitudinal vibrations of automotive helical springs with variable pitch (Flenker and Uphoff, 2005). The second model is based on the concept of the equivalent beam (Haringx, 1949; Wittrick, 1966; Guido et al., 1978; Kobelev, 2014; Michalczyk, 2015b). In the third model, the spring is treated as a spatially curved rod. This model, due to its versatility, is widely presented in the literature, e.g. (Wittrick, 1966; Jiang et al., 1991; Stander and Du Preez, 1992; Becker et al., 2002; Yu and Yang, 2010), however, it should be emphasized that the versatility of this model is accompanied by great complexity and calculation difficulties, meaning that effective application of this model requires the use of numerical methods to solve it, e.g. (Mottershead, 1980; Yildrim, 1999; Taktak et al., 2008). The mentioned complexity and the necessity of using mathematical software whilst solving this model mean that from the engineering application point of view it is easier and faster to use FEM software, with which most of the aforementioned authors compare their results anyway. Moreover, in most of the theoretical studies concerning spring vibrations where the mentioned model is used, the authors do not take into account changes in the lead angle in the vicinity of end coils, assuming that the spring consists only of active coils, which imposes obvious simplifications on the boundary conditions. It has been shown in the paper (Michalczyk, 2015a) that the influence of end-coil shape can be so significant that application of the model treating a spring as a spatially curved rod, but with constant geometrical parameters over the entire length of the wire, may not produce results more accurate than the equivalent beam model, especially with regard to the first frequency of transverse vibrations. The authors of the paper (Liu and Kim, 2009) highlighted the problem of the influence of end-coil shape on the natural longitudinal vibration frequencies of valve springs, proposing a new method for calculating the first transversal frequency, based on the assumption that the torsion stiffness of end coils can be modelled by replacing them with elastic clamping of active coils.

As mentioned above, the model of a modified equivalent Timoshenko beam is utilized in further considerations. The model is widely described in the references cited above and thus only these equations which are necessary to maintain the transparency of derivation are quoted below.

The equation describing natural transverse vibrations of statically axially loaded helical springs can be written in the following form (Haringx, 1949) 


$$
\frac{\partial^{4} \psi}{\partial x^{4}}-\left(\frac{m_{e}}{\beta}+\frac{m_{e} r_{g}^{2}}{\alpha}\right) \frac{\partial^{4} \psi}{\partial x^{2} \partial t^{2}}+\frac{m_{e}^{2} r_{g}^{2}}{\alpha \beta} \frac{\partial^{4} \psi}{\partial t^{4}}+\frac{P}{\alpha}\left(1+\frac{P}{\beta}\right) \frac{\partial^{2} \psi}{\partial x^{2}}+\frac{m_{e}}{\alpha}\left(1+\frac{P}{\beta}\right) \frac{\partial^{2} \psi}{\partial t^{2}}=0
$$

where: $\psi$ - angle of equivalent beam axis deflection resulting only from bending, $x$ - coordinate corresponding to the axis of the non-deflected spring, $P$ - static axial force compressing the spring, $m_{e}$ - linear density of the axially loaded equivalent beam, $\alpha$ and $\beta$ - bending and shearing rigidities of the equivalent beam, respectively, $r_{g}$ - mass radius of gyration of the equivalent beam cross-section

$$
r_{g}=R \frac{\sqrt{2}}{2}
$$

where $R$ denotes a half of the nominal diameter of the spring. A few different formulas describing the bending $\alpha$ and shearing $\beta$ rigidities can be found in the literature. The most recent and accurate formulas were proposed by Krużelecki and Życzkowski (1990), and their approach is utilized in further calculations. These rigidities are expressed in the following form in the cited paper:

$$
\alpha=\frac{2 E J \sin \delta}{2+\nu \cos ^{2} \delta} \quad \beta=\frac{E J \sin \delta}{R^{2}\left(1+\nu \sin ^{2} \delta\right)} \frac{2 \pi n}{\int \sin ^{2} \varphi d \varphi}
$$

where: $E$ - Young's modulus of the spring wire material, $J$ - moment of inertia of the wire cross-section (considerations are restricted to the circular cross-section), $\nu$ - Poisson's ratio, $\delta-$ lead angle of the axially loaded spring, whereas $\varphi$ denotes the angle plotted by the leading radius of a helix between the vector of the transversal force and the arbitrary cross-section of the spring wire. It is assumed for the sake of simplicity that the radius $R$ is constant and equal to half of the nominal spring diameter and the number of working coils $n_{0}$ is also constant, equal to an integer or an integer and a half.

The form of the solution to equation (2.1) depends on whether the searched natural frequency value is higher or lower than the cut-off frequency $\omega_{b}$. Further considerations are limited to the case when the searched value of the first natural transversal vibration frequency is lower than the value of the cut-off frequency $\omega_{b}$, which is expressed in the following form (Michalczyk, 2015a)

$$
\omega_{b}=\sqrt{\frac{\beta}{m_{e} r_{g}^{2}}\left(1+\frac{P}{\beta}\right)}
$$

On the assumptions described above and applying clamped-clamped boundary conditions, the frequency equation may be written as (Michalczyk, 2015a)

$$
\begin{aligned}
0= & \left(-A k_{1}-B k_{1}^{3}\right) C_{1} \sinh \left(k_{1} L\right)+\left(-A k_{1}-B k_{1}^{3}\right) C_{2} \cosh \left(k_{1} L\right) \\
& +\left(A k_{2}-B k_{2}^{3}\right) C_{3} \sin \left(k_{2} L\right)+\left(-A k_{2}+B k_{2}^{3}\right) C_{4} \cos \left(k_{2} L\right)
\end{aligned}
$$

where

$$
\begin{aligned}
A & =\frac{\left(m_{e} \omega^{2} r_{g}^{2}+P\right) \beta+P^{2}}{m_{e} \omega^{2}(\beta+P)} \quad B=\frac{\alpha \beta}{m_{e} \omega^{2}(\beta+P)} \\
k_{1} & =\sqrt{\frac{1}{2}\left[\left(\frac{m_{e}}{\beta}+\frac{m_{e} r_{g}^{2}}{\alpha}\right) \omega^{2}+\frac{P}{\alpha}\left(1+\frac{P}{\beta}\right)\right]\left(-1+\sqrt{1+\frac{4 m_{e}^{2} \omega^{2}\left(-m_{e} \omega^{2} r_{g}^{2}+P+\beta\right) \alpha \beta}{\left[m_{e}\left(\beta r_{g}^{2}+\alpha\right) \omega^{2}+P(\beta+P)\right]^{2}}}\right.} \\
k_{2} & =\sqrt{\frac{1}{2}\left[\left(\frac{m_{e}}{\beta}+\frac{m_{e} r_{g}^{2}}{\alpha}\right) \omega^{2}+\frac{P}{\alpha}\left(1+\frac{P}{\beta}\right)\right]\left(1+\sqrt{1+\sqrt{1+\frac{4 m_{e}^{2} \omega^{2}\left(-m_{e} \omega^{2} r_{g}^{2}+P+\beta\right) \alpha \beta}{\left[m_{e}\left(\beta r_{g}^{2}+\alpha\right) \omega^{2}+P(\beta+P)\right]^{2}}}}\right.}
\end{aligned}
$$




$$
C_{2}=\frac{-\cosh \left(k_{1} L\right)+\cos \left(k_{2} L\right)}{\sinh \left(k_{1} L\right)+\frac{A k_{1}+B k_{1}^{3}}{-A k_{2}+B k_{2}^{3}} \sin \left(k_{2} L\right)} C_{1} \quad C_{3}=-C_{1} \quad C_{4}=\frac{A k_{1}+B k_{1}^{3}}{-A k_{2}+B k_{2}^{3}} C_{2}
$$

whereas $C_{1}$ denotes an arbitrary constant greater than zero.

Solving equation (2.5) numerically, one can obtain the frequencies of natural transverse vibrations of a clamped-clamped spring loaded with the static axial force $P$, which can be expressed as a product of the relative compression of the spring and its compression rigidity.

\section{Determination of a formula allowing direct calculation of the first natural frequency of transverse vibrations}

The results of the analytical model described in the previous section are used in the development of the desired formula. The appropriate equations are implemented in the Maple environment and solved numerically. The following characteristic parameters influencing the natural frequencies are investigated: $d$-wire diameter; $C$ - spring index equal to the ratio of the mean spring diameter $D$ to the wire diameter $d ; \lambda$ - spring slenderness equal to the ratio between the free length $L$ and the mean spring diameter $D ; \delta$ - spring helix angle and $\zeta$ - relative compression of the spring. The range of variation of the listed parameters is presented in Table 1. It is also assumed that the minimum number of active coils is 3 . This limitation results from the fact that, with the decreasing number of coils, the stiffness and inertia properties of the spring become more and more nonuniform, whereas the model of the equivalent Timoshenko beam assumes that these properties are axisymmetric. The assumption of the minimum number of coils means that in the case of a spring with the lowest slenderness $(\lambda=2)$, the highest value of the helix angle $\delta=12^{\circ}$. In the calculations performed, typical material properties of spring steel are assumed: the Young's modulus $E=209000 \mathrm{MPa}$, Poison's ratio $\nu=0.3$ and density $\rho=7800 \mathrm{~kg} / \mathrm{m}^{3}$.

Table 1. The range of variation of spring parameters

\begin{tabular}{|c|c|c|c|c|}
\hline$d[\mathrm{~m}]$ & $C[-]$ & $\delta\left[{ }^{\circ}\right]$ & $\zeta[-]$ & $\lambda[-]$ \\
\hline \hline $0.0005-0.02$ & $3-12.54$ & $3-15$ & $0-0.5$ & $2-10$ \\
\hline
\end{tabular}

Thirty data sets have been used in calculations. These sets were obtained by a combination of 6 values of wire diameter $d(0.0005 \mathrm{~m}, 0.00113 \mathrm{~m}, 0.00258 \mathrm{~m}, 0.00585 \mathrm{~m}, 0.01327 \mathrm{~m}$ and $0.02 \mathrm{~m})$ and 5 values of ratio $C(3,4.29,6.13,8.77$ and 12.54). Each single set was computed for constant values of the wire diameter $d$ and the spring index $C$, while the other parameters varied. The tested values of the helix angle amounted to: $3^{\circ}, 6^{\circ}, 9^{\circ}, 12^{\circ}$ and $15^{\circ}$, whilst the values of slenderness $\lambda$ were equal to $2,4,6,8$ and 10. The tested values of relative compression $\zeta$ were equal to $0,0.1,0.2,0.3,0.4$ and 0.5 when buckling did not appear. When buckling occurred before the relative compression reached 0.5 , the tested values were changed, however, in each set 6 values were always tested. Finally, by solving equation (2.5) numerically, the total number of 4320 results in the form of the first natural frequency of transverse vibrations $f_{T A}$ was obtained.

As mentioned above, it is not possible to derive the desired formula strictly and directly from the analytical model presented in Section 2. Thus, only approximate methods can be applied. Application of an artificial neural network was considered, however, the very large range of output, which in this case was the natural frequency, resulted in very poor performance. In such a case, this method cannot provide results with a sufficiently good agreement with the results of the analytical model within a wide range of variability of these parameters. The formula for predicting the first natural frequency of transverse vibrations of the axially loaded spring $f_{T}$ can be expressed as a function of five parameters, as listed in Table 1

$$
f_{T}=f(d, C, \delta, \lambda, \zeta)
$$


Analysis of the results of numerical calculations shows that, in each of the 30 investigated data sets, the highest value of the first natural frequency $f_{\max }$ for an uncompressed spring $(\zeta=0)$ occurs at the lowest spring slenderness $(\lambda=2)$ and the highest value of the helix angle $\left(\delta=12^{\circ}\right)$. These values are presented graphically in Fig. 1.

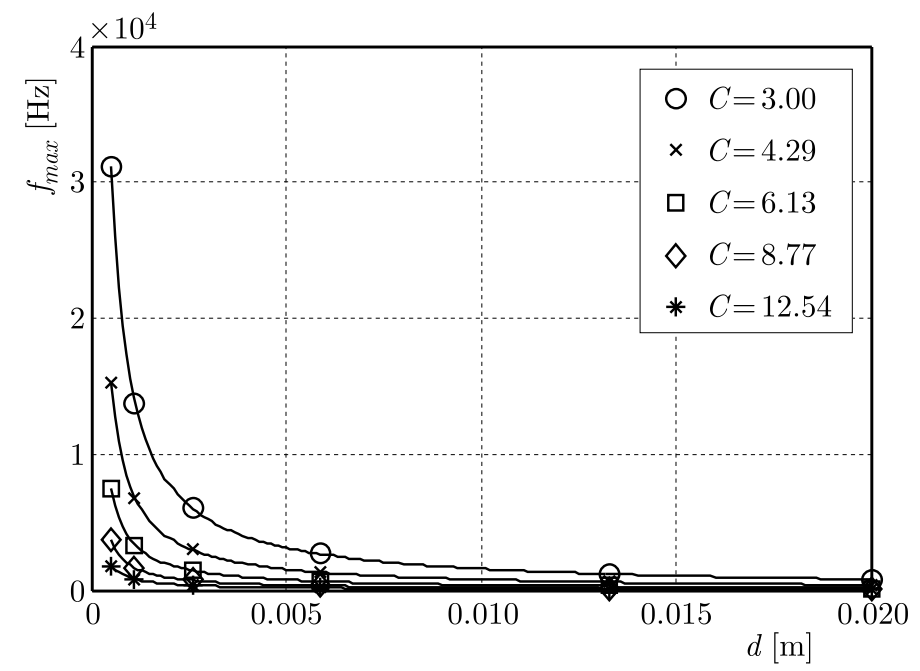

Fig. 1. The maximum $f_{\max }$ values in each of 30 data sets as a function of $d$ and $C$

As shown in Fig. 1, for each tested spring index $C$, the curve fitting procedure was conducted using the least squares method. Each line in Fig. 1 represents a hyperbola that can be expressed in the form

$$
f_{\max }=\frac{a_{0}}{d}
$$

The values of the coefficient $a_{0}$ in equation (3.2), for subsequent values of the spring index $C$, are shown in Table 2.

Table 2. Values of the coefficient $a_{0}$ in relation to the spring index $C$

\begin{tabular}{|c|c|c|c|c|c|}
\hline$n$ & 0 & 1 & 2 & 3 & 4 \\
\hline \hline$C_{n}$ & 3 & 4.29 & 6.13 & 8.77 & 12.54 \\
\hline$a_{0 n}$ & 15.578 & 7.618 & 3.731 & 1.823 & 0.892 \\
\hline
\end{tabular}

It can be noticed that the consecutive values of $C$ and $a_{0}$ create a geometric series. For $C$, the value of the geometric series equals $q=1.43$, while for the coefficient $a_{1}$ the value $q=0.489=$ $1 / 2.044$.

Thus, the maximum frequency $f_{\max }$ depending on the variable $d$ and the fixed values of $C$ equals

$$
f_{\text {max }}=\frac{15.578}{d} \frac{1}{2.044^{n}}
$$

where $n$ is defined by

$$
\frac{C}{3}=1.43^{n}
$$

The formulas above refer to a discrete set consisting of 5 elements. In order to calculate the $f_{\max }$ for any value from the whole range of both $d$ and $C$, it is necessary to make a transformation that $\operatorname{logs}$ both sides of the equation

$$
\log \frac{C}{3}=n \log 1.43
$$


Finally, after proper transformations, we obtain the relationship describing the maximum frequency $f_{\max }$ as a function of the wire diameter $d$ and spring index $C$

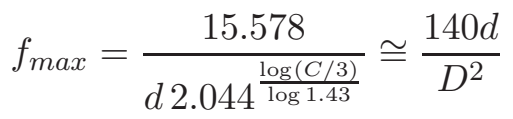

The frequency $f_{T}$ values obtained by means of numerical calculations for all tested combinations of the parameters $d, C, \delta, \lambda$ and $\zeta$ can be divided by appropriate values of $f_{\max }$. As a result, one can obtain the normalized frequencies $f_{n}$ with the same values in each of the 30 data sets, for the same values of $\delta, \lambda$ and $\zeta$

$$
f_{n}=\frac{f_{T}}{f_{\max }}
$$

This significantly simplifies further data analysis. The normalized frequency $f_{n}$ depends on the $\delta, \lambda$ and $\zeta$ values and the relationship between these parameters and the frequency $f_{n}$ has to be determined.

The influence of the change in the helix angle $\delta$ on the normalized frequency $f_{n}$ is highly linear and independent of the other parameters. An increase in the $\delta$ angle is accompanied by a proportional increase in the normalized frequency. The influence of the last two parameters $\lambda$ and $\zeta$ on the normalized frequency $f_{n}$ cannot be considered separately, because a change in the value of one of them changes the character of action of the other. This is illustrated in Fig. 2, which shows the influence of the parameters $\lambda$ and $\zeta$ on the frequency $f_{n}$.

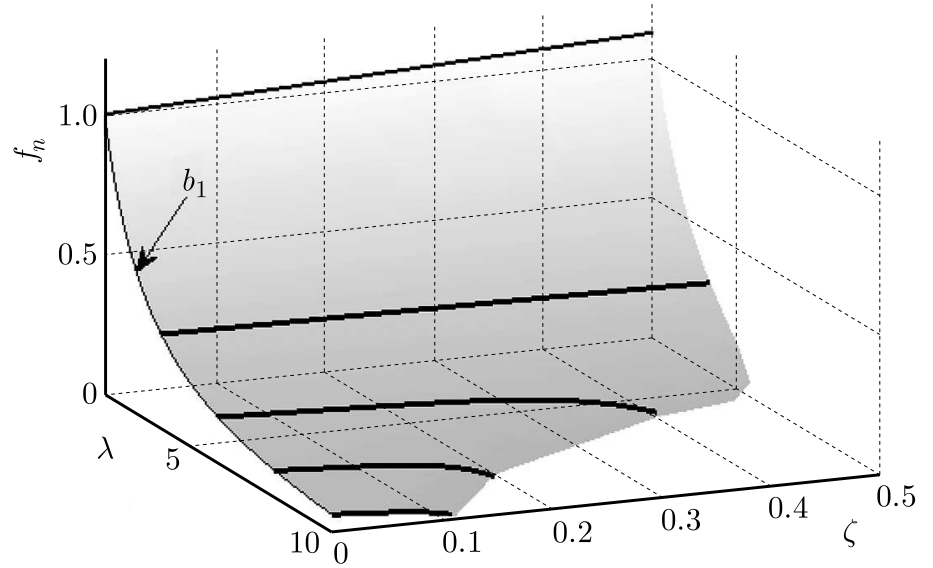

Fig. 2. The normalised frequency $f_{n}$ as a function of the slenderness $\lambda$ and relative compression $\zeta$

Thus, the frequency $f_{n}$ can be presented in the form of a product

$$
f_{n}=c_{1}(\delta) c_{2}(\lambda, \zeta)
$$

where the component $c_{1}$ takes the form

$$
c_{1}=\frac{\delta}{12^{\circ}}
$$

For the following analysis, only frequencies corresponding to $\delta=12^{\circ}$ were considered, since the first factor $c_{1}$ was determined for the maximum frequency that always occurs at an angle of $\delta=12^{\circ}$. The influence of the parameters $\lambda$ and $\zeta$ on the frequency $f_{n}$ is described by the factor $c_{2}$, which is a non-linear function. To obtain such a function, shown in Fig. 3a, two separate functions, $f_{1}(\lambda, \zeta)$ (Fig. 3b) and $f_{2}(\lambda, \zeta)$ (Fig. 3c), are used. 
(a)

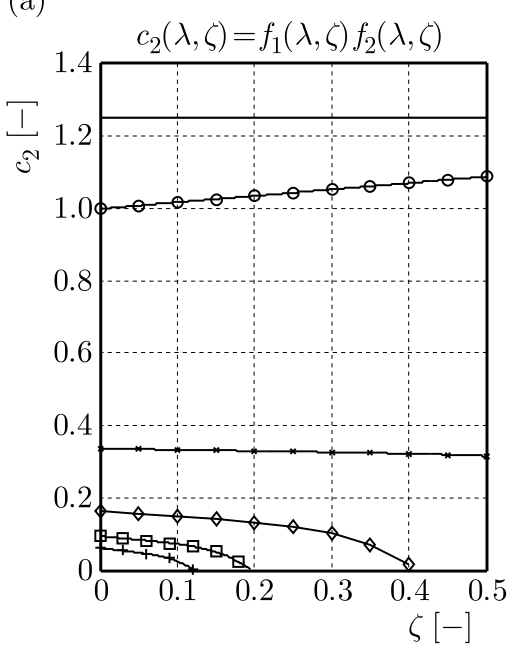

(b)

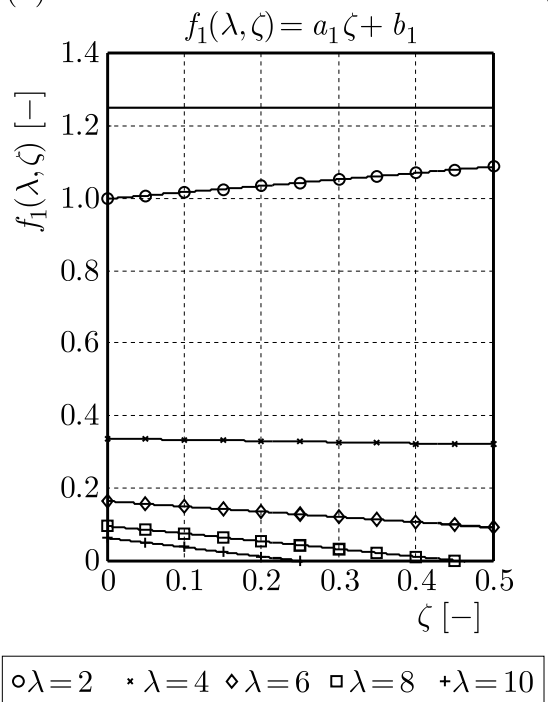

(c)

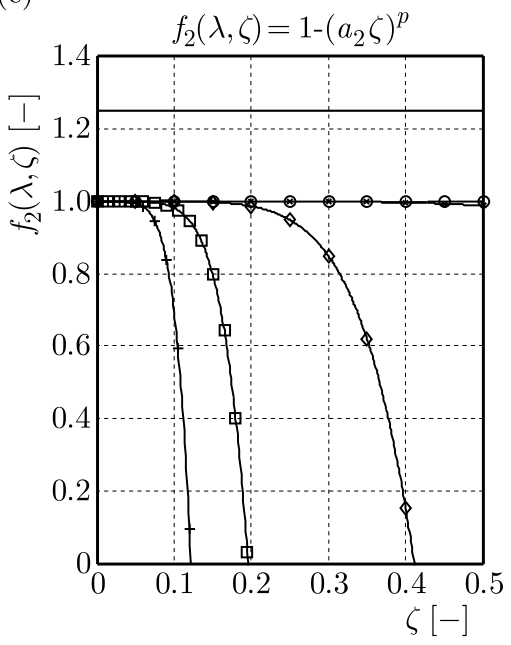

Fig. 3. Description of the coefficient $c_{2}$ as a composition of two separate functions $f_{1}(\lambda, \zeta)$ and $f_{2}(\lambda, \zeta)$

The linear function $f_{1}(\lambda, \zeta)$, which is designated based on the assumption that $f_{1}(\lambda, \zeta)$ is tangent to $c_{2}(\lambda, \zeta)$ at $\zeta=0$, is described by the formula

$$
f_{1}(\lambda, \zeta)=a_{1}(\lambda) \zeta+b_{1}(\lambda)
$$

The proper values of the coefficients $a_{1}(\lambda)$ and $b_{1}(\lambda)$ are presented in Table 3 .

Table 3. Coefficients $a_{1}(\lambda)$ and $b_{1}(\lambda)$ for subsequent values of $\lambda$

\begin{tabular}{|c|c|c|c|c|c|}
\hline$\lambda$ & 2 & 4 & 6 & 8 & 10 \\
\hline \hline$a_{1}(\lambda)$ & 0.18 & -0.04 & -0.15 & -0.20 & -0.26 \\
\hline$b_{1}(\lambda)$ & 1 & 0.3366 & 0.1633 & 0.0952 & 0.0620 \\
\hline
\end{tabular}

The coefficient $a_{1}(\lambda)$ is approximated, with use of the mse method, by a hiperbolic function

$$
a_{1}(\lambda)=\frac{3}{\lambda+2.43}-0.5
$$

The coefficient $b_{1}(\lambda)$, marked in Fig. 2 , is a point of intersection of the linear function $f_{1}(\lambda, \zeta)$ with the vertical axis. For the whole range of $\lambda$ (and $\zeta=0$ ) (Fig. 2), $b_{1}(\lambda)$ is determined with use of Thiele's interpolation curve-fitting method, which in this case gives the most accurate results compared to other methods like spline, polynomial or least squares interpolation, and is described as

$$
b_{1}(\lambda)=\frac{583-9.45 \lambda+0.293 \lambda^{2}}{82.4+95.2 \lambda+73.2 \lambda^{2}}
$$

To decrease the frequency in the final range of compression, the power function $f_{2}$ is introduced

$$
f_{2}(\lambda, \zeta)=1-\left[a_{2}(\lambda) \zeta\right]^{p}
$$

To limit the number of variables in the final formula, thus simplifying it as much as possible, $f_{2}(\lambda, \zeta)$ was fitted with the assumption that the power $p$ is a constant rather than a variable. The results of approximation with use of mse are shown in Table 4. 
Table 4. Coefficients $a_{2}(\lambda)$ and $p$ for subsequent values of $\lambda$

\begin{tabular}{|c|c|c|c|c|c|}
\hline$\lambda$ & 2 & 4 & 6 & 8 & 10 \\
\hline \hline$a_{2}(\lambda)$ & 0.35 & 0.96 & 2.44 & 5.1 & 8.2 \\
\hline$p$ & 6 & 6 & 6 & 6 & 6 \\
\hline
\end{tabular}

To approximate the coefficient $a_{2}(\lambda)$ as a function of $\lambda$, the authors used the logsig function, which resulted in the formula

$$
a_{2}(\lambda)=\frac{12.02}{1+2^{-0.77(\lambda-8.57)}}
$$

Finally, the formula for the transverse frequency $f_{T}$ of the helical spring, after simplifying the fractions, is

$$
f_{T}=\frac{11.667 d \delta}{D^{2}}\left[\frac{583-9.45 \lambda+0.293 \lambda^{2}}{82.4+95.2 \lambda+73.2 \lambda^{2}}+\left(\frac{3}{\lambda+2.43}-0.5\right) \zeta\right]\left[1-\left(\frac{12.02 \zeta}{1+2^{-0.77(\lambda-8.57)}}\right)^{6}\right]
$$

The frequencies $f_{T}$ obtained with developed formula (3.15) were compared with the frequencies $f_{T A}$ obtained by numerical solving of the analytical model. The relative difference $\Delta_{f}$ between the frequencies $f_{T A}$ and $f_{T}$ can be defined as follows

$$
\Delta_{f}=\left|\frac{f_{T A}-f_{T}}{f_{T A}}\right| \cdot 100 \%
$$

The value of the relative difference $\Delta_{f}$ depends only on the spring slenderness $\lambda$ and relative compression $\zeta$. The results of calculations of $\Delta_{f}$ in the whole working range of $\lambda$ and $\zeta$ is shown in Fig. 4.

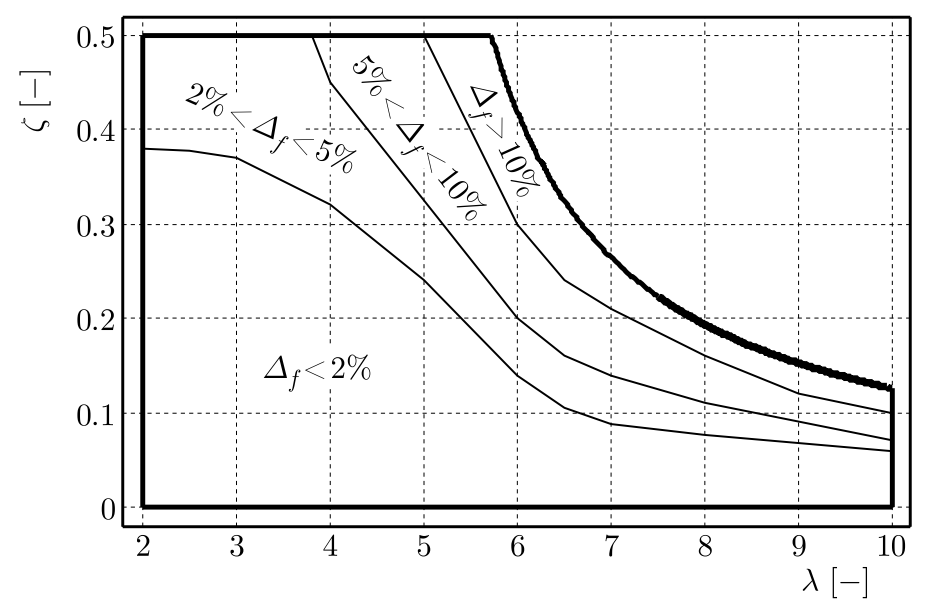

Fig. 4. The relative difference $\Delta_{f}$ as a function of the spring slenderness $\lambda$ and relative compression $\zeta$

Analysing Fig. 4, it can be noticed that the range of the $\lambda$ and $\zeta$ parameters, for which the relative difference $\Delta_{f}$ is small, is very wide. This difference, however, increases with higher values of $\lambda$ and $\zeta$ which are accompanied by frequencies approaching to zero. The curve limiting the plot in Fig. 4 from the top-right corner represents the line of spring buckling obtained on the basis of the analytical model. The result is that even a small absolute difference between the frequencies $f_{T A}$ and $f_{T}$ gives a high value of $\Delta_{f}$. 


\section{Comparison of the results obtained using the derived formula with those obtained using FEM and experiments}

Relationship (3.15) was developed on the basis of data obtained from the analytical model, in which the helical spring was modelled using the concept of an equivalent Timoshenko beam. This model has axially symmetrical properties regarding inertia and stiffness and, thus, does not take into account the effects associated with the spring wire curvature and the real boundary conditions. In order to assess the accuracy of the developed formula, its results for selected

Table 5. Comparison of the first natural frequencies of transverse vibrations obtained from FEM $\left(f_{I}\right.$ and $\left.f_{I I}\right)$ with the results of developed formula (3.15)

\begin{tabular}{|c|c|c|c|c|c|c|c|c|c|}
\hline No. & $\begin{array}{c}d \\
{[\mathrm{~mm}]}\end{array}$ & $\begin{array}{c}D \\
{[\mathrm{~mm}]}\end{array}$ & $\begin{array}{c}h \\
{[\mathrm{~mm}]}\end{array}$ & $n$ & $\delta_{0}$ & $\zeta$ & $\begin{array}{c}f_{I}(\mathrm{FEM}) \\
{[\mathrm{Hz}]}\end{array}$ & $\begin{array}{c}f_{I I}(\mathrm{FEM}) \\
{[\mathrm{Hz}]}\end{array}$ & $\begin{array}{c}f_{T}(3.15) \\
{[\mathrm{Hz}]}\end{array}$ \\
\hline 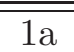 & \multirow{3}{*}{10} & \multirow{3}{*}{100} & \multirow{3}{*}{40} & \multirow{3}{*}{5} & $\overline{77.256}$ & 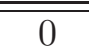 & 83.056 & 85.907 & 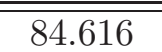 \\
\hline $1 b$ & & & & & 7.256 & 0.25 & 84.638 & 87.757 & 88.366 \\
\hline $1 \mathrm{c}$ & & & & & 7.256 & 0.5 & 86.335 & 89.839 & 92.114 \\
\hline $2 \mathrm{a}$ & \multirow{3}{*}{10} & \multirow{3}{*}{100} & \multirow{3}{*}{40} & \multirow{3}{*}{5.25} & 7.256 & 0 & 78.475 & 80.669 & 78.971 \\
\hline $2 \mathrm{~b}$ & & & & & 7.256 & 0.25 & 80.132 & 81.899 & 82.405 \\
\hline $2 \mathrm{c}$ & & & & & 7.256 & 0.5 & 81.885 & 83.167 & 85.836 \\
\hline $3 \mathrm{a}$ & \multirow{3}{*}{10} & \multirow{3}{*}{100} & \multirow{3}{*}{40} & \multirow{3}{*}{5.5} & 7.256 & 0 & 73.101 & 76.940 & 73.852 \\
\hline $3 \mathrm{~b}$ & & & & & 7.256 & 0.25 & 74.75 & 77.591 & 76.983 \\
\hline $3 \mathrm{c}$ & & & & & 7.256 & 0.5 & 76.611 & 78.186 & 80.11 \\
\hline $4 a$ & \multirow{3}{*}{10} & \multirow{3}{*}{100} & \multirow{3}{*}{40} & \multirow{3}{*}{5.75} & 7.256 & 0 & 68.023 & 72.347 & 72.038 \\
\hline $4 b$ & & & & & 7.256 & 0.25 & 69.306 & 72.836 & 72.038 \\
\hline $4 c$ & & & & & 7.256 & 0.5 & 70.747 & 73.530 & 74.873 \\
\hline $5 \mathrm{a}$ & \multirow{3}{*}{10} & \multirow{3}{*}{100} & \multirow{3}{*}{20} & \multirow{3}{*}{10} & 3.642 & 0 & 42.476 & 42.843 & 42.471 \\
\hline $5 \mathrm{~b}$ & & & & & 3.642 & 0.25 & 43.451 & 43.851 & 44.354 \\
\hline $5 c$ & & & & & 3.642 & 0.5 & 44.534 & 44.979 & 46.235 \\
\hline $6 a$ & \multirow{3}{*}{10} & \multirow{3}{*}{100} & \multirow{3}{*}{40} & \multirow{3}{*}{10} & 7.256 & 0 & 28.299 & 28.447 & 28.477 \\
\hline $6 \mathrm{~b}$ & & & & & 7.256 & 0.25 & 26.090 & 26.178 & 27.764 \\
\hline $6 c$ & & & & & 7.256 & 0.5 & 21.785 & 21.815 & 26.721 \\
\hline $7 \mathrm{a}$ & \multirow{3}{*}{10} & \multirow{3}{*}{100} & \multirow{3}{*}{60} & & 10.8125 & 0 & 20.501 & 20.525 & 20.586 \\
\hline $7 \mathrm{~b}$ & & & & 10 & 10.813 & 0.2 & 14.343 & 14.358 & 16.725 \\
\hline $7 \mathrm{c}$ & & & & & 10.8125 & 0.3 & 7.361 & 7.398 & 12.848 \\
\hline $8 a$ & & & & & 7.256 & 0 & 13.816 & 13.823 & 13.815 \\
\hline $8 \mathrm{~b}$ & & & & & 7.256 & 0.2 & 9.650 & 9.668 & 11.224 \\
\hline $8 c$ & 10 & 100 & 40 & 15 & 7.256 & 0.3 & 4.789 & 4.796 & 8.622 \\
\hline $8 d$ & & & & & 7.256 & 0.32 & 2.561 & 2.568 & 7.707 \\
\hline $9 \mathrm{a}$ & & & & & 7.256 & 0 & 6.912 & 6.915 & 6.907 \\
\hline $9 \mathrm{~b}$ & & & & & 7.256 & 0.2 & 4.812 & 4.814 & 5.612 \\
\hline $9 \mathrm{c}$ & 5 & 100 & 40 & 15 & 7.256 & 0.3 & 2.355 & 2.357 & 4.311 \\
\hline $9 \mathrm{~d}$ & & & & & 7.256 & 0.32 & 1.203 & 1.226 & 3.854 \\
\hline $10 \mathrm{a}$ & & & & & 7.256 & 0 & 27.571 & 27.588 & 27.629 \\
\hline $10 \mathrm{~b}$ & & & & & 7.256 & 0.2 & 19.429 & 19.437 & 22.447 \\
\hline $10 \mathrm{c}$ & 20 & 100 & 40 & 15 & 7.256 & 0.3 & 10.178 & 10.205 & 17.243 \\
\hline $10 \mathrm{~d}$ & & & & & 7.256 & 0.32 & 6.293 & 6.334 & 15.414 \\
\hline
\end{tabular}

spring parameters and deflections were compared with the results of FEM analyses, in which the spring was modelled as a spatially curved bar. The analyses were performed in the ANSYS environment. The model was built using BEAM 189 elements, which take into account the effects 
of shearing and rotational inertia of cross-sections. In the analyses concerning springs subjected to a non-zero static deflection, the perturbation method was utilized, taking into account large deflection effects. A comparison of FEM analyses results with those obtained from equation (3.15) is presented in Table 5.

It can be noticed that the results of FEM analyses and those of formula (3.15) are generally in very good compliance, especially for low and moderate relative compression. The differences between the obtained results become larger for high relative compression values and high values of slenderness. It can also be noticed that the $f_{I}$ and $f_{I I}$ frequencies obtained for each case from FEM analyses have very similar values, regardless of the number of coils. The largest differences between corresponding the $f_{I}$ and $f_{I I}$ values were obtained for $n=5.75$.

(a)

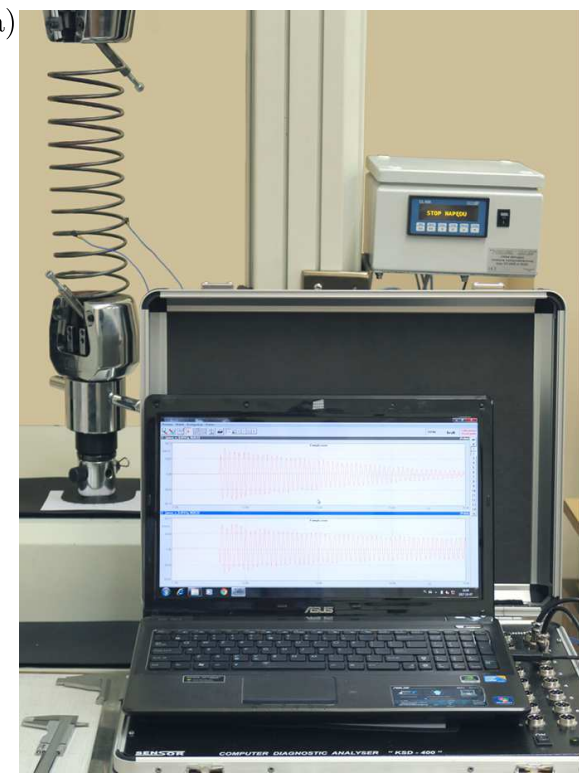

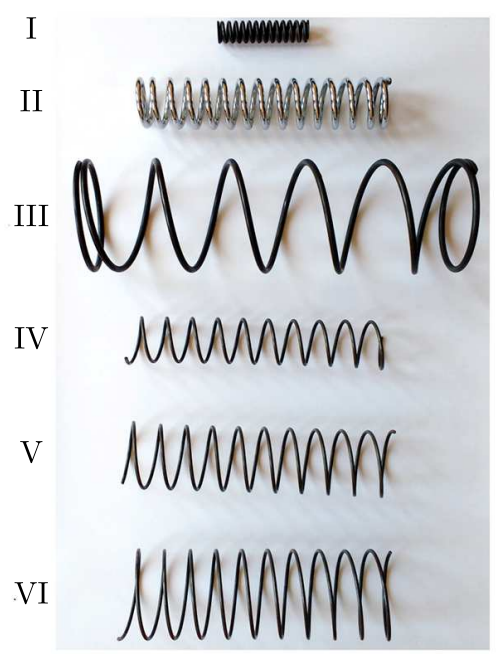

(c)

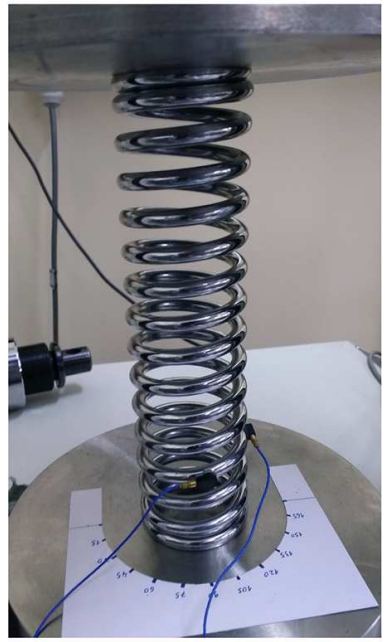

Fig. 5. Experimental test rig with one of the tested springs, two PCB322 accelerometers mounted on it, and a KSD-400 universal vibration analyzer (a), six investigated springs (b) and one of the compression springs supported on flat plates (c)

Experimental tests using six different springs were also performed. The test rig is shown in Fig. 5a, and the tested springs are shown in Fig. 5b. Three of these springs (I, II and III in Fig. 5b) were industrial compression springs with partially square and ground ends, whilst three others $(I V, V$ and $V I)$ were specially prepared springs with open ends and all coils active. The first three springs were placed between flat supporting plates as shown in Fig. 5c, and the last three springs were fixed in special grippers, visible in Fig. 5a. For each of the tested springs, the tests were conducted at two values of relative compression. The first three springs were initially compressed in order to achieve the adequate gripping friction force. The last three springs were not compressed initially as they were fixed using specially prepared grippers. The second value of relative compression was the same in all the cases and equalled 0.25. A non-zero initial condition on the transverse displacement was imposed to the middle coils, and natural transverse vibration time courses were recorded using two PCB Piezotronics model 352C22 miniature accelerometers and a Sensor KSD-400 vibration analyser. Each test was conducted at six different directions of the initial displacement condition, changing by every $30^{\circ}$ in order to find the first two natural frequencies of transverse vibrations. Table 6 presents a comparison of the results of experimental tests with those obtained using equation (3.15).

It can be noticed that the largest differences between the results of experiments and those of formula (3.15) occur for springs $I, I I$ and $I I I$, whilst for the last three springs the obtained results are very consistent. The greater discrepancy in the first case is results from the effect 
Table 6. Comparison of the results of experimental tests with those obtained using equation (3.15) for six investigated springs

\begin{tabular}{|c|c|c|c|c|c|c|c|c|c|}
\hline No. & $\begin{array}{c}d \\
{[\mathrm{~mm}]}\end{array}$ & $\begin{array}{c}D \\
{[\mathrm{~mm}]}\end{array}$ & $\begin{array}{c}h \\
{[\mathrm{~mm}]}\end{array}$ & $n$ & $\delta_{0}$ & $\zeta$ & $\begin{array}{c}f_{I} \text { (exp.) } \\
{[\mathrm{Hz}]}\end{array}$ & $\begin{array}{c}f_{I I} \text { (exp.) } \\
{[\mathrm{Hz}]}\end{array}$ & $\begin{array}{c}f_{T}(3.15) \\
{[\mathrm{Hz}]}\end{array}$ \\
\hline$\overline{I_{a}}$ & \multirow{2}{*}{4} & \multirow{2}{*}{20.5} & \multirow{2}{*}{7.65} & \multirow{2}{*}{12.75} & \multirow{2}{*}{$6.77^{\circ}$} & $\overline{0.1}$ & 171.38 & $\bar{~} 172.86$ & $\overline{180.5}$ \\
\hline$I_{b}$ & & & & & & 0.25 & 168.63 & 170.36 & 170.9 \\
\hline$I I_{a}$ & \multirow{2}{*}{7} & \multirow{2}{*}{49} & \multirow{2}{*}{19.3} & \multirow{2}{*}{14.5} & \multirow{2}{*}{$7.15^{\circ}$} & 0.1 & 35.7 & 37.24 & 40.2 \\
\hline$I I_{b}$ & & & & & & 0.25 & 33.21 & 35.38 & 34.04 \\
\hline$I I I_{a}$ & \multirow{2}{*}{7} & \multirow{2}{*}{113} & \multirow{2}{*}{80} & \multirow{2}{*}{5} & \multirow{2}{*}{$12.7^{\circ}$} & 0.05 & 25.26 & 26.34 & 33.626 \\
\hline$I I I_{b}$ & & & & & & 0.25 & 28.18 & 28.71 & 33.665 \\
\hline$I V_{a}$ & \multirow{2}{*}{4} & \multirow{2}{*}{50} & \multirow{2}{*}{28.6} & \multirow{2}{*}{10.4} & \multirow{2}{*}{$10.32^{\circ}$} & 0 & 32.24 & 32.33 & 31.934 \\
\hline$I V_{b}$ & & & & & & 0.25 & 23.55 & 23.79 & 23.985 \\
\hline$V_{a}$ & \multirow{2}{*}{4} & \multirow{2}{*}{70} & \multirow{2}{*}{28.6} & \multirow{2}{*}{10.5} & \multirow{2}{*}{$7.41^{\circ}$} & 0 & 21.11 & 21.69 & 21.032 \\
\hline$V_{b}$ & & & & & & 0.25 & 19.97 & 20.15 & 20.077 \\
\hline$V I_{a}$ & \multirow{2}{*}{4} & \multirow{2}{*}{88} & \multirow{2}{*}{28} & \multirow{2}{*}{10.5} & \multirow{2}{*}{$5.78^{\circ}$} & 0 & 15.52 & 15.75 & 15.876 \\
\hline$V I_{b}$ & & & & & & 0.25 & 15.79 & 16.02 & 16.049 \\
\hline
\end{tabular}

of end-coils. The end-coils were not taken into account in calculations and, as a result, higher values of frequencies were obtained from equation (3.15) than those from experiments.

\section{Conclusions}

The formula developed in the paper, allowing the first natural frequency of transverse vibrations of clamped-clamped and axially loaded with a static force helical springs to be predicted, is, according to the authors' knowledge, the first such a formula in the available literature. It allows calculation of both the first natural frequency of a spring as well as determination of the relative compression at which the spring buckling will occur. The formula can be widely used among designers of dynamical systems, where helical steel springs are the most commonly used type of elastic joints, significantly facilitating their calculations. The results obtained on the basis of the developed relationship demonstrate very good agreement in a wide range of the spring geometric parameters and its relative compression with the results of the analytical model utilizing the Timoshenko equivalent beam concept, those of FEM analyses and the performed experiments. An important advantage of the developed formula is the lack of necessity to use expensive software and knowledge of complex analytical or numerical models in order to determine the first natural frequency of transverse vibrations.

\section{Acknowledgements}

The authors acknowledge the financial support of AGH University of Science and Technology, Faculty of Mechanical Engineering and Robotics, project No. 11.11.130.174.

\section{References}

1. Becker L.E., Chassie G.G., Cleghorn W.L., 2002, On the natural frequencies of helical compression springs, International Journal of Mechanical Sciences, 44, 825-841

2. Cieplok G., 2009, Verification of the nomogram for amplitude determination of resonance vibrations in the run-down phase of a vibratory machine, Journal of Theoretical and Applied Mechanics, 47, 295-306 
3. Flenker Ch., Uphoff U., 2005, Efficient valve-spring modelling with MBS valve-train design, MTZ Worldwide, 66, 946-950

4. Guido A.R., Della Pietra L., Della Valle S., 1978, Transverse vibrations of helical springs, Meccanica, 13, 2, 90-108

5. HARIngX J.A., 1949, On highly compressible helical springs and rubber rods, and their application for vibration-free mountings, II, Philips Research Reports, 4, 49-80

6. Jiang W., Jones W. K., Wang T. L., Wu K. H., 1991, Free vibrations of helical springs, Transactions of ASME, 58, 222-228

7. Kobelev V., 2014, Effect of static axial compression on the natural frequencies of helical springs, Multidiscipline Modeling in Materials and Structures, 10, 379-398

8. KruŻelecki J., ŻyCZKowski M., 1990, On the concept of an equivalent column in the problem of stability of compressed helical springs, Ingenieur-Archiv, 60, 367-377

9. Lee J., Thompson D. J., 2001, Dynamic stiffness formulation, free vibration and wave motion of helical springs, Journal of Sound and Vibration, 239, 297-320

10. LiU H., Kim D., 2009, Effects of end coils on the natural frequency of automotive engine valve springs, International Journal of Automotive Technology, 10, 413-420

11. MichalcZYK K., 2015a, Analysis of lateral vibrations of the axially loaded helical spring, Journal of Theoretical and Applied Mechanics, 53, 3, 745-755

12. MichalczyK K., 2015b, Dynamic stresses in helical springs locally coated with highly-damping material in resonant longitudinal vibration conditions, International Journal of Mechanical Sciences, 90, 53-60

13. Mottershead J.E., 1980, Finite elements for dynamical analysis of helical rods, International Journal of Mechanical Sciences, 22, 267-283

14. Sapiński B., Snamina J., Jastrzębski Ł., Staśkiewicz A., 2011, Laboratory stand for testing self-powered vibration reduction systems, Journal of Theoretical and Applied Mechanics, 49, 1169-1181

15. Stander N., Du Preez R. J., 1992, Vibration analysis of coil springs by means of isoparametric curved beam finite elements, Communications in Applied Numerical Methods, 8, 373-383

16. TAKtak M., Dammak F., Abid S., Haddar M., 2008, A finite element for dynamic analysis of a cylindrical isotropic helical spring, Journal of Materials and Structures, 3, 641-658

17. WitTrick W.H., 1966, On elastic wave propagation in helical springs, International Journal of Mechanical Sciences, 8, 25-47

18. YILDRIM V., 1999, An efficient numerical method for predicting the natural frequencies of cylindrical helical springs, International Journal of Mechanical Sciences, 41, 919-939

19. Yu A. M., YAng C. J., 2010, Formulation and evaluation of an analytical study for cylindrical helical springs, Acta Mechanica Solida Sinica, 23, 1, 85-94 